\title{
La teoría de la audiencia en el contexto de la entrevista sociolingüística. La influencia del género de los interlocutores en el manejo de los turnos y la temática * Audience Theory in the Context of the Sociolinguistic Interview. Interlocutors' Gendered Roles in the Management of Turns and Topics
}

Sandra Soler Castillo **

Recibido: 15 de agosto del 2013 / Aprobado: 23 de septiembre del 2013

\section{Resumen}

En este texto se trata el problema de la audiencia y su influencia en la interacción discursiva en contexto de entrevista. Se plantea cómo las características sociales de los interlocutores, en especial el género, influyen en la estructuración de la interacción en lo referente al manejo de turnos y temáticas de conversación. Siguiendo los principios de la sociolingüística y el análisis del discurso, se analiza un corpus de treinta relatos (entrevistas), cada uno de una hora, tomados por los investigadores del Instituto Caro y Cuervo. Se concluye que el manejo de turnos y las temáticas de conversación no están relacionados solo con la cooperación dentro de la conversación o con la lucha por mantener el poder, como hasta ahora se ha explicado, sino también con la identificación de los interlocutores entre sí y el conocimiento de los estilos del otro. Así, los participantes de la conversación tienden a variar su estilo conversacional según su audiencia, pero también tienden a adaptarse a la manera de hablar del otro.

Palabras clave: audiencia, turnos, temas, género, entrevista

\section{Abstract}

This paper looks into audiences and their influence on discursive interaction in the context of interviewing. The study draws on the idea that interlocutors' social features, especially gender, play a role in the interaction construction based on turn taking and topic choice. A 30-interview corpus is analyzed drawing on sociolinguistics and discourse analysis. Each interview lasts an hour and data was collected by 'Instituto Caro y Cuervo' researchers. Findings demonstrate that turn taking and topic choice are not simply related to talk cooperation or to issues of power. It is argued that both turn taking and topic choice are also related to conversational styles and to how much interlocutors know each other. Thus participants tend to adjust their conversational styles according to audiences as they also adapt to other's ways of speaking.

Keywords: Audience, turns, topics, gender, interview

\footnotetext{
Este artículo es una proyección investigativa de la tesis doctoral Discurso y género en historias de vida. Una investigación de relatos de hombres y mujeres en Bogotá, del programa de doctorado en Lingüística y Comunicación de la Universidad de Barcelona, sustentada en agosto del 2003.

** Doctora en Lingüística y Comunicación de la Universidad de Barcelona, docente de la Universidad Distrital, directora del Doctorado Interinstitucional en Educación de la Universidad Distrital. Investigadora en las líneas de discurso, discriminación y escuela. Correo electrónico: stsolerc@udistrital.edu.co.
} 


\section{Introducción}

La entrevista constituye sin duda una de las herramientas centrales de la investigación cualitativa en las ciencias sociales y humanas y en particular en educación. Con el auge de los enfoques hermenéutico-narrativos, comprender las dinámicas discursivas de la interacción verbal implica detenerse en las condiciones que ayudan a construir las subjetividades de los hablantes. En ese sentido, el género se constituye en una categoría relevante que permite explicar fenómenos como el poder, la solidaridad o la cooperación y ayuda a identificar los estilos de conversación de hombres, mujeres y otros géneros que pugnan cada vez más por su reconocimiento.

El objetivo de este trabajo es demostrar cómo la audiencia determina o contribuye a la estructuración de la interacción discursiva; más específicamente, cómo el género de los interlocutores afecta el sistema de turnos y el desarrollo de las temáticas de conversación, además de resaltar la importancia metodológica que tiene en el contexto de la investigación.

El interés en este tema surgió de la investigación tendiente a hallar las diferencias de género en la manera de hablar de hombres y mujeres en Bogotá (2004), y aunque este tema no se tuvo en cuenta para el informe final de investigación, se propuso para desarrollarlo en ulteriores trabajos dada su importancia y la influencia que podría tener en los análisis de corpus, pues al indagar sobre el manejo de turnos y temas se encontraron notables diferencias en los relatos de hombres y mujeres. Nuestra hipótesis es que el estilo conversacional no solo está modificado por la audiencia, las características del hablante o los factores relativos al discurso y al contexto, sino que también estos mismos factores también se determinan entre sí en el interior del estilo mismo. Así, por ejemplo, los factores que propone Moreno Fernández (2009), relativos al discurso (temas y tipo de conversación), estarían también determinados por las características de la audiencia.

\section{Referentes teóricos}

El problema de la audiencia tiene una amplia tradición en los estudios del lenguaje, pero sus orígenes quizá se encuentren en Bajtín y su teoría del dialogismo. Fue este autor quien sin duda llamó la atención sobre cómo todo discurso está orientado hacia otro: el oyente, incluso cuando este no se encuentra presente en la conversación. A comienzos del siglo XX, Bajtín señalaba que "En la realidad, cada enunciación - un discurso, una conferencia, etc.- está dirigida a un oyente, es decir, a su comprensión y a su respuesta [...], a su consenso y disenso, en otras palabras a la percepción valorativa del oyente - al auditorio" (1929/1993, p. 250). Sin embargo, como todos sabemos, estas tempranas teorías de Bajtín, que habrían podido influir bastante e incluso cambiar el curso de la lingüística, solo se conocieron en la década de los años setenta del siglo XX. De esta manera, en América y Europa, el problema de la audiencia aparece en el campo de los estudios del lenguaje por la vía del desarrollo de la ciencia lingüística y en particular de la sociolingüística.

La sociolingüística define su campo de estudio en lo relativo a la variación y las variedades lingüísticas. La primera, referida a cómo hacen uso las personas de ciertos elementos lingüísticos, en lugar de otros del mismo nivel, sin que esto suponga cambios semánticos; variación que si depende de factores sociales, como la clase social, la edad o el género, recibe el nombre de variación sociolingüística. Las segundas —variedades lingüísticas—, según las define Moreno Fernández, se refieren a los "conjuntos de elementos o de patrones lingüísticos asociados a factores externos, sean contextos situacionales, sean ámbitos profesionales, sean grupos sociales, sean áreas geográficas" (2009, p. 86). Dentro de estas variedades se encuentran las lenguas, los dialectos, los sociolectos, las jergas y los estilos o registros.

La variación sociolingüística ha sido bastante estudiada y ha logrado un amplio grado de desarrollo en el mundo entero. La variación estilística, en cam- 
bio, como bien lo señalara Bell al formular su teoría de la audiencia, "no ha sido adecuadamente explicada en la teoría sociolingüística” (1984, p. 145). Hoy, después de décadas de análisis, aún quedan algunos interrogantes por resolver. Es nuestro interés en estas líneas revisar algunos de los aspectos relativos al estilo y su determinación -o mejor construccióna partir de la audiencia.

El estilo y la variación estilística tienen que ver con los usos lingüísticos determinados a partir de la situación y el contexto comunicativo. Para algunos autores, el estilo representa un código diferenciado que puede variar de acuerdo con la situación; piénsese en las teorías de Bell, Giles o Halliday. Para otros, por el contrario, no se trata de la selección de uno u otro código, sino de una gradación a partir de un estilo básico, denominado estilo vernáculo. El exponente más importante de esta teoría es Labov, para quien el estilo depende del grado de atención que preste el hablante al discurso.

De esta manera, se tendría una escala que iría del habla informal (vernáculo) al habla formal, con posibilidad de que aparezca también el habla espontánea (habla menos formal en el contexto de entrevista). La diferencia fundamental entre estas teorías radica, en principio, en sobre quién se pone mayor énfasis como determinante de la variación estilística: sobre el hablante (Labov) o sobre el interlocutor (Bell, Giles). La variación estilística, además de estar determinada por factores relativos al hablante y al interlocutor, también depende de las características de la situación comunicativa misma.

Respecto al estilo, dos teorías han tenido más desarrollo y explican mejor el tema: la teoría de la audiencia (Bell, 1984) y la teoría de la acomodación (Giles, 1984). Al proponer estas teorías los autores intentaban explicar cómo las personas varían su estilo conversacional dependiendo no solo del — como lo había señalado Labov (1972) — grado de atención prestado al discurso, o del hecho de que la situación misma sea más o menos formal, sino además de la re- lación que se da entre los interlocutores (audiencia). Bell sostiene que el estilo es, en esencia, la respuesta del hablante a su audiencia, en la que este se acomoda antes que nada a su interlocutor. Para el autor, el diseño de la audiencia es un tema más amplio que el simple cambio de código en el que se seleccionan unas unidades en lugar de otras; implica todos los niveles posibles de elección lingüística, incluye cambios tan amplios como pasar de una lengua a otra, de un dialecto a otro o de un registro a otro.

También abarca la forma de los actos de habla, la elección de pronombres, el uso de títulos honoríficos y los cambios cuantitativos de estilo. Bell define la audiencia como "la gente que escucha las oraciones del hablante" (1984, p. 145), pero para él no se trata de entes pasivos, pues la audiencia responde, interpreta o critica y, por tanto, ayuda a construir la interacción.

Giles (1984), por su parte, en la teoría de la acomodación propone que las personas utilizan diversas estrategias comunicativas para adaptarse a las situaciones y al habla de sus interlocutores (convergencia), así como para alejarse de ellas (divergencia), dependiendo de los intereses del hablante y del oyente, que van desde la simple aprobación social hasta la defensa de la identidad. La teoría de la acomodación no es una teoría propiamente dicha sobre la variación estilística, aunque se use para explicarla; se trata de una teoría de corte psicosocial que busca dar cuenta de los procesos cognoscitivos producidos entre la percepción del contexto y las conductas comunicativas y la acomodación de los hablantes a dichos contextos (Moreno Fernández, 2009, p. 155).

Aunque las dos teorías resalten la importancia del interlocutor, la teoría de la audiencia tiene un carácter más sociolingüístico. Bell diferencia entre variación sociolingüística y variación estilística; la primera es interindividual y la segunda intraindividual. El estilo sería una proyección de la dimensión sociolingüística, en la cual ciertos usos lingüísticos se adaptan a ciertas posibilidades sociolingüísticas 
que ofrece la comunidad, pero en el plano intraindividual.

La relevancia de estas teorías radica en la importancia que tienen en el momento de solucionar una de las cuestiones más complejas de la metodología de investigación social: cómo acceder a los datos lingüísticos (narrativos) y cómo evitar que la presencia del o la entrevistadora influya de modo directo en ellos; lo que se conoce como paradoja del observador. Son muchas las páginas escritas en torno a esta problemática. Para superarla, se ha intentado proponer temas en los que la emoción prime para que el o la entrevistada se haga menos consciente de la situación y del lenguaje. También se ha sugerido interactuar con la persona en repetidas ocasiones antes de la entrevista formal, o incluso acompañarla en sus distintas actividades para crear familiaridad y hablarle de temas cotidianos.

A pesar de todo lo escrito, es nuestro parecer que aún no se ha puesto el punto final a este debate. Quedan todavía factores por analizar en la interacción comunicativa en el contexto de la entrevista; uno de ellos, y el que nos ocupa aquí, es la influencia del género de la persona del entrevistador. Los hombres han sido históricamente los investigadores por excelencia. De todos es sabido que la mujer solo ha sido tenida en cuenta como informante y como investigadora en épocas recientes. Son los hombres quienes han determinado qué preguntar, a quién, cómo y dónde. El propósito de este artículo es demostrar cómo el estilo conversacional está profundamente marcado por las características sociales de la audiencia, más exactamente por el género del interlocutor o la interlocutora.

\section{Metodología}

A partir de los principios de la sociolingüística y el análisis del discurso se comenzará por examinar cómo se da el manejo de turnos conversacionales, para demostrar cómo varían los estilos dependiendo del género del entrevistador. Luego se pasará al aná- lisis de las temáticas de conversación. Se plantea un análisis básico de tipo cuantitativo.

El corpus. El material que sirve de base a este análisis lo constituyen treinta relatos semilibres, de una hora de duración cada uno, tomados por el equipo de investigadores e investigadoras del Instituto Caro y Cuervo (Montes, 1997). En principio se trataba de que los o las informantes hablaran durante una hora sobre los temas que desearan, sin que el entrevistador o la entrevistadora interrumpieran para preguntar o proponer temas. Pero esto no fue posible siempre, en bastantes ocasiones los encuestadores y las encuestadoras debieron realizar preguntas. Para el análisis que aquí se plantea es importante tener en cuenta cómo estuvo constituido el grupo de encuestadores. En total fueron 6 encuestadores: 3 hombres y 3 mujeres. Los hombres realizaron 16 de las 30 entrevistas y las mujeres las 14 restantes. Ambos grupos encuestaron hombres y mujeres. Los hombres encuestaron 8 hombres y 8 mujeres y las mujeres siete hombres y 7 mujeres.

\section{Resultados}

A continuación se presentan los resultados con base en el manejo de turnos y temas de conversación.

\section{Turnos}

Se entiende por turno el tiempo que se conceden los hablantes unos a otros dentro de una conversación. En la figura 1 se pueden ver algunos datos generales del corpus en cuanto a los turnos.

Figura 1. Manejo general de turnos

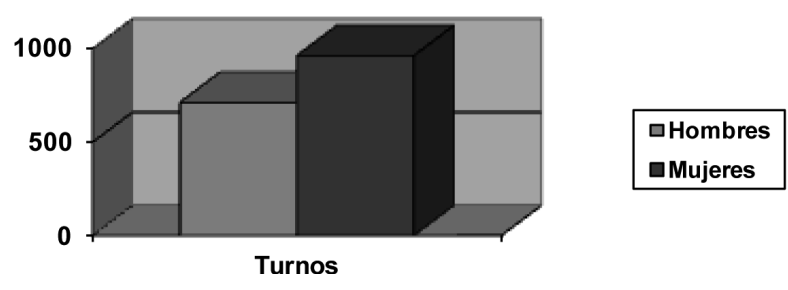

Fuente: elaboración propia. 
La información que aparece en la figura 1 es la que generalmente se ofrece en los estudios de las interacciones discursivas y el manejo de turnos (usos generales sin importar información adicional). Así, a simple vista podríamos concluir que en el habla de Bogotá, en el contexto de entrevistas, las mujeres emplean más turnos que los hombres. ${ }^{1}$ Pero es importante explicar por qué se da este hecho y qué tipo de información se trasmite en cada turno, es decir, cómo se dan verdaderamente los intercambios comunicativos. Al analizar con más detalle se encuentran evidencias como las que se presentan en la figura 2.

Figura 2. Tipo de interacción

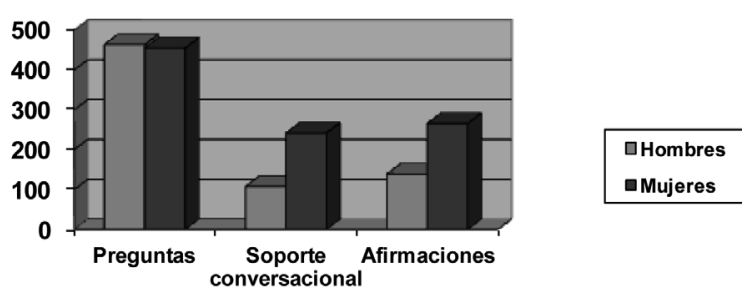

Fuente: elaboración propia.

En la figura 2 se observa que los entrevistadores realizaron más o menos el mismo número de preguntas, pero hubo más soporte conversacional de las entrevistadoras mujeres que de los hombres. Se entiende por soporte conversacional el mantenimiento de la función fática mediante el uso de formas de asentimiento del tipo aja, um ja o sí. Resulta entonces interesante investigar cómo se dio este proceso. ¿Ocurrió lo mismo al tratarse de entrevistas mixtas (entrevistadora/entrevistado, entrevistador/entrevistada) o en parejas del mismo género (entrevistadora/ entrevistada, entrevistador/entrevistado)? ¿Varió el

1 Cestero (1994) llega a similares conclusiones en su estudio sobre la alternancia de turnos en lengua española. La autora encuentra que, en general, las mujeres emplean más turnos en las conversaciones que los hombres, y explica este hecho por la mayor tendencia de las mujeres a cumplir con las normas de cortesía de la interacción. comportamiento de encuestadores y encuestadoras al entrevistar hombres y mujeres, y variaron estos su comportamiento a su vez al ser entrevistados y entrevistadas por hombres o mujeres? A continuación se revisará este hecho.

La manera de entrevistar de hombres y mujeres presenta divergencias. Los hombres establecen las entrevistas siguiendo el patrón pregunta-respuestapregunta. Las mujeres, por el contrario, se involucran más en la entrevista y su realización obedece más a la forma dialógica pregunta-respuesta-comentariopregunta. En la figura 3 se presenta el comportamiento de los y las informantes de acuerdo con el género del encuestador.

Figura 3. Manejo de turnos con encuestador hombre

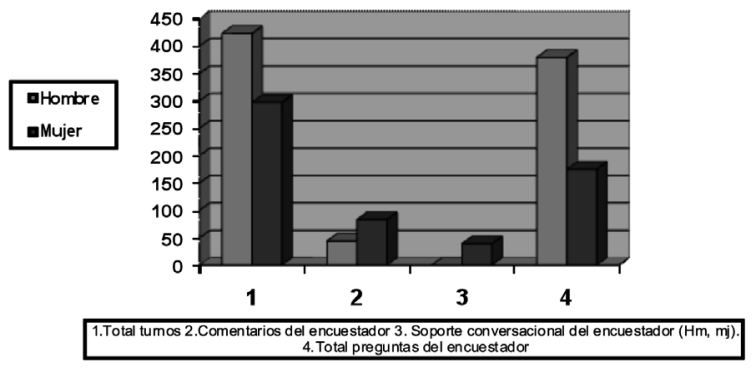

Fuente: elaboración propia.

En la figura 3 se observan diferencias fundamentales en el comportamiento de los encuestadores y encuestados. En las entrevistas realizadas por hombres se emplearon más turnos cuando entrevistado y entrevistador fueron hombres que cuando hombres entrevistaron a mujeres (423/297), y los encuestadores intervienen menos para hacer comentarios (44/83) o para asentir (0/39), pero realizan más preguntas a los informantes hombres que a las mujeres (379/175), sea para ampliar los temas, o para proponer otros nuevos. De lo que se concluye que en la interacción en contexto de entrevista cuando el entrevistador es hombre, se establece una interacción mayor con el entrevistado cuando este también es 
hombre. En este caso hay mayor número de preguntas relativas al tema de conversación, pero al entrevistar mujeres, los entrevistadores se adaptan más a su estilo: asienten más y realizan más comentarios a las respuestas. Veamos algunos ejemplos: ${ }^{2}$

- Entrevistador hombre con informante hombre:

ENC: Muy bien, entonces comience con su relato.

INF: Desde septiembre he estado pasando papeles en varias empresas y todo, pero no me sale trabajo. Me sale trabajo por quince o un mes, ya, ya se acaba el trabajo. No, no consigo empleo por ningún lao. Toes hotica estoy desempleao.

ENC: ¿Qué más ha hecho?

INF: Pues, horitica naa, buscando trabajo, recomendando trabajos, a ver si me salen. Porque no, no consigo trabajo por ningún lao.

ENC: ¿En qué le gustaría trabajar?

INF: Pues en lo que salga, cualquier cosa.

Obsérvese cómo el encuestador establece la entrevista a partir de preguntas y no expresa su punto de vista ante las respuestas. Se trata de avanzar a partir de generar más preguntas. Compárese las diferencias con el comportamiento de las encuestadoras mujeres (figura 4).

Figura 4. Manejo de turnos con encuestadora mujer

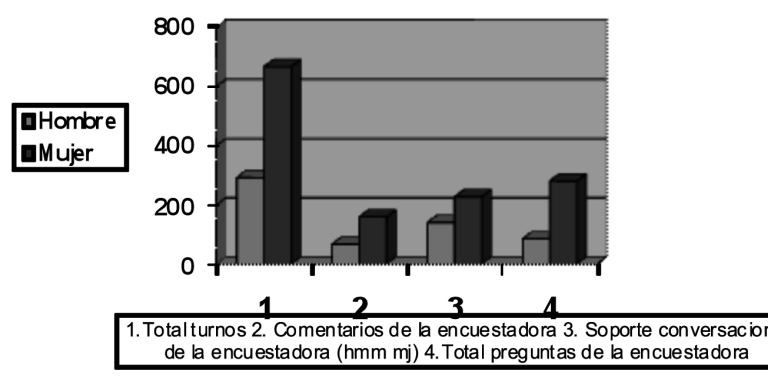

Fuente: elaboración propia.

2 Se sigue la transcripción propuesta en Montes et al. (1997).
En las encuestas realizadas por entrevistadoras se observa que hay mayor interacción cuando se entrevista a las mujeres, siendo la diferencia bastante considerable (292/666). Esto se debió fundamentalmente a que las encuestadoras hicieron más afirmaciones como contribución a la conversación (67/159) y dieron más soporte conversacional cuando entrevistaron a sus congéneres que cuando entrevistaron a hombres (140/227); también realizaron más preguntas relativas al tema tratado (85/280). Veamos algunos ejemplos:

- Entrevistadora mujer con informante hombre:

ENC: Muy bien, entonces podemos empezar.

INF: Bueno, como... por ejemplo, aquí... la enseguridá es bastante... pesada.

ENC: $\mathrm{Mj}, \mathrm{mj}$

INF: Y no hay a quién acudir, no hay quién haga nada pues por... colaboración en eja inseguridá. Ni loj mismoj vecinos, ni loj mismoj papases, porque no hay gente de afuera.

ENC: $\mathrm{Mj}, \mathrm{mj}$.

INF: Es gente del mijmo, barrio. Y eso es lo que lo, lo intranquiliza a uno aquí, nada más; porque lo que's servicios: agua, luz, todo es correpto. Pero la inseguridá es lo más, que lo intranquiliza a uno.

Obsérvese cómo la encuestadora ofrece soporte conversacional al informante asintiendo mediante expresiones del tipo $M j$, $m j$, que lo invitan a continuar la conversación, o al hacer afirmaciones y comentarios relativos al tema de conversación, como se observa en el ejemplo siguiente:

- Entrevistadora mujer con informante hombre

INF: Un padre de familia con cinco chinoj y loj manda ayá y que lej den el desayunito, ¿cuánto se’stá horrando para... comprarle un par de tenis o alguna vaina?

ENC: Cierto.

INF: Pero... ya él entrega, ahi muere’so; ahí muere ya eso. 
ENC: Sí, hay niños que van a la escuela ni que...

INF: En ayunas.

ENC: Ni siquiera con una agua de panela.

INF: N... nada, nada, ¿sí? (tose) m... Ahora... hay muchas, mucha formaj, de poder... que el gobierno colabore, en... asuntos de... m... el principiante; familias demasiado pobres. Ahora usté ¿como, cómo ve, la... l'asunto de la... la de Armero? Uno que conoció, que fue Armero, es una cosa desastrosa, ver uno, en lo que quedó.

ENC: Ahora.

Nótese cómo la entrevista se desarrolla en forma de diálogo, en el que tanto encuestador como encuestado ofrecen sus puntos de vista sobre el tema. Como conclusión en cuanto a los turnos podemos decir que, como se había presentado en la hipótesis, las personas (locutor/interlocutor) varían el estilo conversacional según su audiencia. Tanto hombres como muje res se involucran más al entrevistar a sus congéneres, y estos a su vez varían sus comportamientos discursivos al ser entrevistados por unos y otras. Ambos grupos tienden a adaptarse al estilo conversacional del otro, sea asintiendo más o menos o haciendo más o menos comentarios, dependiendo del género del entrevistado.

\section{Manejo de temas}

El manejo de temas está directamente relacionado con la propuesta y el desarrollo de los turnos, como se observa en las figuras 5 y 6 .

Figura 5. Manejo de temas con encuestador hombre

\section{口Hombre $\square$ Mujer}

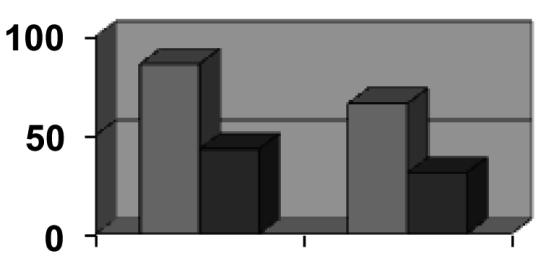

1

1. Total temas tratadospor los informantes hombres y mujeres 2 . Temas propuestos por el encuestador para hombres y mujeres

Fuente: elaboración propia.

En la figura 5 se observa que los hombres trataron mayor cantidad de temas cuando fueron entre- vistados por sus congéneres (86) que cuando los entrevistaron mujeres (69), como lo muestra la figura 6.

Figura 6. Manejo de temas con encuestadora mujer

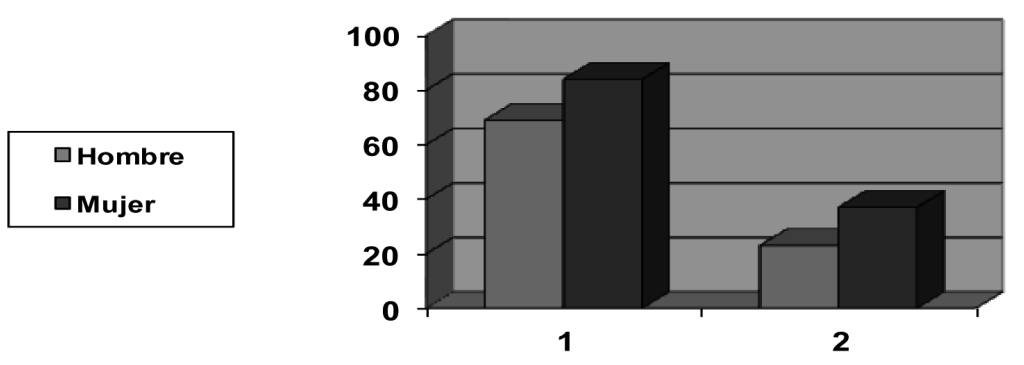

1. Total temas tratados por informantes hombres y mujeres 2 . Temas propuestos porel encuestador para hombres y mujeres.

Fuente: elaboración propia. 
Las mujeres presentan este mismo comportamiento: cuando fueron entrevistadas por sus congéneres trataron 84 temas, y cuando fueron entrevistadas por hombres tan solo 43. En general, se puede afirmar que los encuestadores hombres propusieron más temas cuando entrevistaron a sus congéneres (66) que cuando entrevistaron mujeres (31); las encuestadoras hicieron lo mismo, aunque con menor diferencia: cuando entrevistaron a hombres propusieron 23 temas, mientras que cuando entrevistaron mujeres propusieron 37 .

Juntando estos datos con los de las figuras anteriores de los turnos, se observa que la interacción varía de acuerdo con el género de los participantes. En la entrevista entre hombres hubo mayor desarrollo de temas en términos de las preguntas relativas a los temas realizadas por los congéneres (379), que permitieron ampliarlos y profundizarlos, que cuando entrevistaron mujeres (175). Las encuestadoras mostraron igual comportamiento: cuando entrevistaron hombres plantearon 85 preguntas y cuando entrevistaron mujeres propusieron 280 preguntas de ampliación y complementación. Veamos algunos ejemplos de estas interacciones de los y las encuestadoras con sus congéneres:

- Encuestador hombre, informante hombre. Tema: recuerdos de la ciudad

ENC: Más penitencia, ¿no?

INF: ¿ Si?, el hombre tenía por ahi como unos... cincuenta años, tal vez, en ese tiempo, ¿no?, claro que ya estaba... él era muy gordo, entonces lo, lo fatigaba la, la, la subida, ¿no?, entonces me... yo echaba adelante y me tocaba esperar hasta llegar allá. Ya bajando pues sí, ya nos veníamos en... bajábamos en funicular, ¿sí? Pero eso iba mucha gente en ese tiempo. De allá sí se divisaba lo que era Bogotá, lo com.. los pueblitos retiraos, ¿no? También conocí a, a la, el reci... ese... ese vaino del... de, de Techo, ¿no?, el aeropuerto de Techo lo conocí; eso era muy bonito.

ENC: Y había un hipódromo también...

INF: Bastante grande, ¿sí? Hipódromo, ni un eh...

\section{ENC: Hipódromo de Techo.}

INF: ¡Ah...! eso fue después. Eso fue después, mucho después ¿Sí?

ENC: El primer aeropuerto fue el de Techo...

INF: El primer aeropuerto fue el de Techo, ¿sí? Y ahí, pues ya...

ENC: Y los aviones eran...

INF: Los aviones eran aviones de esos viejos, ¿no? Había unos que los llamaban... no me acuerdo como era que se llamaban esos aviones, ¿no?, que era los más importantes, ¿no?, los más de lujo, ¿no? Eh... no recuerdo en el momento preciso, ¿no?, ¿sí? Pero ya le digo: cuando eso eh... caía Lanza, estaba Avianca, estaba Arca... bueno, después ya vino Taxader, ¿no?, ya Avianca, si...

\section{ENC: ¿Y Satena?}

INF: Satena, también existía, ¿sí? Esa viajaba únicamente era a los Llanos, ¿no?

ENC: A los territorios...

En la interacción anterior se observa cómo en torno al tema de los cambios en Bogotá a lo largo del tiempo, el entrevistador interviene constantemente ampliando el tema al introducir sus propios recuerdos de la ciudad y asuntos particulares como el hipódromo, los aviones y las aerolíneas hacia donde se dirige la entrevista.

- Encuestadora mujer con informante mujer, tema: drogadicción:

ENC: ¿Tú cómo crees que se acabaría el problema de la drogadicción en este barrio?

INF: Pues... habiendo m... bastante... m... policía, que hubiera unos los CAI, estuvieran pendiente del barrio, que si vieran muchachos, que los corrigieran ¿sí? Que’so no se debería'ser, que.. invitarlos pues que a trabajar, a colaborar, en cambio de andar en... esos pasos; m... ¿ ande podría decir yo?

ENC: ¿Con tus hermanitos qué harías tú para que eyos no... no salgan a la caye, no corran el mismo riesgo de la otra gente? Como tú prácticamente 
los cuidas con tu hermana ¿entonces son como la mamá, no?

INF: Sí señora. Pues... mis hermanos caji no, eyos no les gusta la caye es... de pequeñitos nunca les ha gustao la caye; más bien son deicaos al estudio, y a trabajar; pero no, eyos no, eyos a, a ece nos ponemos hablar con mi mamá y eyos icen que... quèyos no les gustaría seir los mismos pasos que los... muchachos de por ahi de la cuadra porque. . . eyos han visto muchos casos y no que'yos nos quisiean seguir esa misma... esa misma... ritmo de eyos. Que'yos más bien les gustaría m... estudiar y ayudarle a mi mamá; salir aelante y... m... Que por ejemplo... a mis hermanos no le, no me gustaría que'yos vieran tanto programa violento; que [...] en la teleisión siempre pre... pues prejentan unos programas que son... violentos, que e guerra. Pues eso es muy malo para eyos porque eyos... siguen... pues siguen... en... piensan que'so es mejor para eyos y no... en cambio de ver programas para niños y...

ENC: ¿O sea, que tú no estás de acuerdo con... con los programas de la televisión?

INF: Pues hay unos que sí, hay otros que no, porque hay unos educativos y... y hay otros que son... de mucha guerra. Y...

ENC: Cuéntame de los programas que te gustan, háblame de lo que te gusta de la televisión.

Nótese que la encuestadora propone el tema de cómo acabar con la drogadicción en el barrio, al cual la informante responde de manera general, e inmediatamente la encuestadora lleva el tema al ámbito familiar de la informante quien responde encuadrándolo en relación con la televisión y su influencia, para de esta manera introducir otra temática.

\section{Consideraciones finales}

En un sentido amplio y para finalizar podemos señalar que la comunicación, la interacción, el discurso y la discursividad constituyen en la actualidad temas centrales dentro de los estudios del lenguaje y las ciencias sociales y humanas. Es en la interacción donde los humanos logran, más allá de la simple codificación-decodificación, emisión-recepción de mensajes dar sentido a la experiencia comunicativa. El lenguaje no es un simple repertorio de signos del cual echamos mano para comunicar ideas, es una construcción social dinámica, recursiva y creativa. Es un fenómeno de dos caras, pero no como lo señalara Saussure, sino como lo destacara Bajtín: "cada enunciación presupone, para su realización, la existencia no solo de un hablante sino también de un oyente" (1993, p. 245). Sujetos y discursos que se determinan y construyen mutuamente en dinámicas discursivas situadas.

Pensar la interacción verbal en este sentido posibilita una nueva visión de los diferentes intercambios discursivos que supone, si se quiere, una visión más constructiva del lenguaje en la que los sentidos se van creando en cada una de las secuencias comunicativas y donde la presencia del otro está siempre allí. Cargada de connotaciones construidas también socialmente: el otro con una condición de género, étnica, económica, física, intelectual, etc.

Reconocer ese otro y reconocer la posibilidad de construirnos nosotros mismos como la otra cara del fenómeno, el frente a frente, podría potenciar procesos sociales más equitativos y éticos en los que las diferencias fueran vistas como ventajas y factores de enriquecimiento. En el campo de la educación una mirada de este tipo podría sin duda ayudar a mejorar los intercambios sociales y comunicativos.

Por otra parte, centrándonos en la investigación que acabamos de presentar, concluimos que las personas tienden a variar su estilo conversacional según su audiencia, pero también tienden a adaptarse a la manera de hablar del otro. Así, al analizar fenómenos tan interesantes como el manejo de los turnos en la conversación, se concluye que estos no estarían relacionados solo con la cooperación dentro de la conversación, o con la lucha por mantener el poder, como hasta ahora se ha explicado, sino también con la identificación de los interlocutores entre sí y el co- 
nocimiento de los estilos del otro, sea el manejo de turnos, sea con los temas tratados.

El estudio de la audiencia y su determinación del tipo de estilo sería también de especial ayuda para explicar algunos de los malentendidos e interferencias que se dan en la interacción en que se mezclan diversos estilos comunicativos, como el caso de las conversaciones entre hombres y mujeres, donde las segundas acusan a los primeros de no escucharlas, de no interesarse por sus temas de conversación, al no asentir constantemente, no establecer el canal fático con respuestas mínimas como, sí, $\mathrm{mm} m j$, es verdad, te escucho, o al no hacer preguntas mínimas que demuestren interés, y los primeros las acusan de repetitivas y de interrumpir con excesiva frecuencia, justamente cuando ellas preguntan y se interesan por el tema; o los malentendidos producidos en las interacciones interétnicas, como lo señala Tannen (1981, 1984) al hablar de las interacciones entre judíos y norteamericanos.

Desde el punto de vista metodológico, resulta trascendental que al realizar investigaciones en las que haya entrevistas, encuestas o cuestionarios, se tenga en cuenta el género del entrevistador para evitar al máximo posible los cruces de estilos y la variación que estos producen, como queda demostrado en estas líneas. En la actualidad, se tiende a recomendar que los hombres entrevisten a sus congéneres y, de igual manera, las mujeres a las mujeres. Esta práctica se está realizando en proyectos de la importancia de PRESEEA (Proyecto para el Estudio Sociolingüístico del Español de España y América).

\section{Referencias}

Bajtín, M. (1993). La construcción de la enunciación. En A. Silvestri y G. Blank, Bajtín y Vigotski: la organización semiótica de la conciencia. Barcelona: Anthropos.
Bell, A. (1984). Language Style as Audience Design. Language in Society, 13, 145-204.

Cestero, A. (1994). Alternancia de turnos de habla en lengua española: la influencia del sexo y la edad en los interlocutores. Pragmalingüística, 2, 123-150.

Duranti, A. (1986). The Audience as Co-author: an Introduction. Text, 6 (3), 239-247.

Giles, H. (1984). The Dynamics of Speech Accommodation. International Journal of the Sociology of Language, 46 (special issue).

Goodwin, C. (1986). Audience Diversity, Participation and Interpretation. Text, 6 (3), 283-316.

Labov, W. (1972). Sociolinguistic Patterns. Philadelphia: UPP.

Montes Giraldo, J. J. et ál. (1997). El español hablado en Bogotá. Bogotá: Instituto Caro y Cuervo.

Moreno Fernández, F. (2009). Principios de sociolingüística y sociología del lenguaje. Barcelona: Ariel.

Soler Castillo, S. (2004). Discurso y género en historias de vida. Una investigación de relatos de hombres y mujeres en Bogotá. Bogotá: Instituto Caro y Cuervo.

Tannen, D. (1981). New York Jewish Conversational Style. International Journal of the Sociology of Language, 30, 133-139.

Tannen, D. (1984). Conversational Style: Analyzing Talk among Friends. Norwood: Ablex.

Tannen, D. (2008). La relatividad de las estrategias lingüísticas. Repensar el poder y la solidaridad en el género y la dominación. La Manzana de la Discordia, 3, (2), 91-105. 